\title{
FUNCIONALIDAD FAMILIAR Y SOBRECARGA EN CUIDADORES DE NIÑOS CON EPILEPSIA
}

\section{FAMILY FUNCTIONALITYAND OVERLOAD IN CAREGIVERS OF CHILDREN WITH EPILEPSY}

Andrea González-Araujo* y Juan R. Ruiz-Carlo

Departamento de Educación e Investigación en Salud, Unidad de Medicina Familiar N. ${ }^{\circ}$ 2, Hospital General de Zona, Instituto Mexicano del Seguro Social, Irapuato, Guanajuato, México

RESUMEN: Antecedentes: La epilepsia es la discapacidad motora más frecuente en el mundo, contribuyendo de forma directa en la funcionalidad familiar y en la sobrecarga del cuidador primario. Objetivo: Determinar la asociación entre la funcionalidad familiar y la sobrecarga del cuidador en familias de pacientes pediátricos con epilepsia. Material y métodos: Estudio observacional y de corte transversal en 100 familias de pacientes pediátricos de entre 5 y 16 años de edad con diagnóstico de epilepsia, adscritos al Hospital General de Zona/ Unidad de Medicina Familiar 2, durante el 2019, a quienes se les aplicó el test de funcionalidad familiar FF-SIL y el test de Zarit al cuidador primario. Resultados: Se utilizó la prueba estadística de Chi cuadrada de Pearson, donde resultó el valor de $\mathrm{p}<0.001$, siendo estadísticamente significativa la asociación entre la funcionalidad familiar y la sobrecarga del cuidador primario. Conclusiones: Existe una asociación estadísticamente significativa entre la funcionalidad familiar y la sobrecarga del cuidador en familias de pacientes pediátricos con epilepsia.

Palabras clave: Funcionalidad familiar. Sobrecarga del cuidador. Epilepsia.
ABSTRACT: Background: Epilepsy is estimated to be the most frequent motor disability globally, contributing directly to the familiar dynamics and the burden of the primary caregiver. Objective: To determine the association between the family functionality and the primary caregiver overload of pediatric patients with an epilepsy diagnosis. Material and methods: An observational and cross-sectional study has been done through a non-probabilistic sampling selected at convenience, using descriptive statics in 100 families of pediatric patients between 5 and 16 years old with a diagnosis of Epilepsy ascribed at Hospital General de Zona/ Unidad de Medicina Familiar 2 during 2019, to whom was applied FF-SIL test that evaluates familiar functionality and Zarit test which evaluates primary caregiver overload. Results: Pearson's chi-square statistical test was used, with value of $\mathrm{p}<0.001$, being statistically significant the association between the family functionality and the primary caregiver overload. Conclusions: There is a significant association between the family functionality and the primary caregiver overload in the families of pediatric patients with a diagnosis of epilepsy.

Key words: Family functionality. Caregiver burden. Epilepsy.
Correspondencia:

*Andrea González-Araujo

E-mail: andygonz200@gmail.com
Fecha de recepción: 22-12-2020

Fecha de aceptación: 28-05-2021
Disponible en internet: 11-08-2021

Rev Mex Med Fam. 2021;8:56-61 DOI: 10.24875/RMF.20000211

2007-9710 / C 2021 Federación Mexicana de Especialistas y Residentes en Medicina Familiar. Publicado por Permanyer. Este es un artículo open access bajo la licencia CC BY-NC-ND (http://creativecommons.org/licenses/by-nc-nd/4.0/). 


\section{INTRODUCCIÓN}

La epilepsia es una enfermedad neurológica crónica muy común en la infancia ${ }^{1}$, de acuerdo con la Organización Mundial de la Salud (OMS) existen 50 millones de personas en el mundo con esta enfermedad. La incidencia de la epilepsia en países desarrollados se encuentra entre 42 y 61 por cada 100,000 habitantes, cifras que suelen acercarse al doble, o bien la sobrepasan en países en vías de desarrollo. En nuestro país la prevalencia estimada es de entre 180 y 400 por 100,000 habitantes en la población infantil. Cada año se reportan de 400 a 800 casos nuevos por cada 100,000 niños ${ }^{2}$.

La OMS define la epilepsia como una enfermedad cerebral crónica que afecta a personas de todo el mundo y se caracteriza por convulsiones recurrentes ${ }^{3}$. La presencia de una enfermedad crónica representa un reto para la familia y trae consecuencias positivas o negativas, ya que la familia constituye la fuente principal de apoyo en la atención a las personas enfermas ${ }^{4}$, debido a esto es importante considerar un manejo integral en este grupo de familias. La detección de una enfermedad crónica en uno de los miembros de la familia trae un cambio radical en el interior del individuo y su familia ${ }^{5}$. Cuando el enfermo es un paciente pediátrico, se genera mayor estrés en los padres, quienes en la mayoría de los casos ocupan el papel de cuidador primario; esto puede incrementar la carga de trabajo para el cuidador primario, lo que también puede modificar la funcionalidad familiar.

La funcionalidad familiar es determinante en la conservación de la salud o la aparición de enfermedades en sus integrantes e involucra aspectos como: la adaptación, el crecimiento, la participación, la resolución y el afecto ${ }^{6}$. Parece ser que los cuidadores con una mejor función familiar presentan mejor salud mental. Se puede decir que una familia es funcional cuando los roles de todos los miembros están establecidos, de modo que todos los integrantes contribuyen de forma equitativa para el bienestar colectivo ${ }^{7}$.

El concepto de carga se remonta a los años sesenta ${ }^{8}$. La sobrecarga es el grado en que la persona cuidadora percibe que el cuidado ha influido sobre diferentes aspectos de su vida social, personal y económica ${ }^{4}$, ya que el cuidador se ve sometido a un estrés permanente ${ }^{9}$, por ser el responsable del cuidado del enfermo.

Cuidador primario o cuidador informal es la persona que asume la responsabilidad absoluta del enfermo, ayudándole a realizar las actividades que no puede llevar a cabo, sin recibir una retribución económica ${ }^{10}$. En la mayoría de los casos este papel lo ocupa alguno de los integrantes de la familia.

\section{MATERIAL Y MÉTODOS}

Se realizó un estudio de tipo observacional, analítico y de corte transversal con muestreo no probabilístico seleccionado a conveniencia en 100 familias de pacientes pediátricos de entre 5 y 16 años de edad con diagnóstico de epilepsia, adscritos al Hospital General de Zona/ Unidad de Medicina Familiar 2 (HGZ/UMF 2), Irapuato, Guanajuato, durante el 2019, que aceptaron participar en el estudio con previa firma de consentimiento informado. Se incluyeron las familias de pacientes que tuvieran un tiempo de diagnóstico de epilepsia mayor a un año y que estuvieran en seguimiento por consulta externa de medicina familiar en el HGZ/UMF 2. Se excluyeron pacientes hospitalizados, con diagnóstico concomitante de parálisis cerebral o con una crisis familiar aguda.

Se tomaron en cuenta las siguientes variables internas: funcionalidad familiar $y$ sobrecarga. Como variables sociodemográficas se tomaron en cuenta: edad, sexo, estado civil y escolaridad. Finalmente, los indicadores fueron: cohesión, adaptabilidad, armonía, comunicación, permeabilidad, afectividad y roles. 
Se aplicaron dos test en los integrantes de las familias de pacientes pediátricos con diagnóstico de epilepsia:

- El cuestionario FF-SIL, que evalúa funcionalidad familiar, con una sensibilidad del $90 \%$, una especificidad del $70 \%$ y una confiabilidad superior al 0.75 . Consta de 14 reactivos de 7 indicadores: cohesión, armonía, comunicación, permeabilidad, afectividad, roles y adaptabilidad. Con opciones de respuesta y puntuación en escala de Likert (casi nunca $=1$ punto, pocas veces $=2$ puntos, a veces $=3$ puntos, muchas veces $=4$ puntos y casi siempre $=5$ puntos), con una calificación mínima de 14 puntos y una máxima de 70 puntos. Es una escala que permite clasificar la funcionalidad familiar en: funcional (70 a 57 puntos), moderadamente funcional (56 a 43 puntos), disfuncional (42 a 28 puntos) y severamente disfuncional (27 a 14 puntos) $)^{11}$.

- El test de Zarit del cuidador primario, diseñado para valorar la vivencia subjetiva de sobrecarga sentida por el cuidador principal. Explora los efectos negativos sobre el cuidador en distintas áreas de su vida: salud física, psíquica, actividades sociales y recursos económicos. Cuenta con opciones de respuesta de formularios dicotómicos y con puntuación en escala de Likert de 5 puntos $(0=$ nunca, 1 = casi nunca, $2=$ a veces, $3=$ bastantes veces y 4 = casi siempre). El instrumento tiene una calificación mínima de 22 puntos y una máxima de 110 puntos; a mayor puntuación, mayor nivel de sobrecarga. Cuenta con una escala que permitirá clasificar al cuidador en: $<47=$ no sobrecarga, 47- $55=$ sobrecarga leve $y>55=$ sobrecarga intensa. Tiene una sensibilidad del $100 \%$, una especificidad del $90.5 \%$ y una confiabilidad de $0.90^{12}$.
De acuerdo con lo estipulado en la Ley General de Salud, en materia de investigación para la salud (título segundo, capítulo I, artículo 17, sección 1), nuestro estudio corresponde a investigación sin riesgo. Los datos solo son conocidos por los investigadores, siendo confidenciales. El estudio fue aprobado por el comité de ética en investigación y el comité local de investigación en salud. Los participantes fueron informados verbalmente sobre los objetivos del estudio y se les solicitó su autorización por escrito en una carta de consentimiento informado, basándose en la Ley General de Salud, en la declaración de Helsinki y en el código de Núremberg.

Se utilizó estadística descriptiva: medidas de tendencia central y dispersión para las variables cuantitativas, porcentajes y frecuencias para las variables cualitativas.

Para determinar la asociación entre la funcionalidad familiar y la sobrecarga del cuidador primario se utilizó estadística inferencial mediante la prueba de Chi cuadrada de Pearson $\left(c^{2}\right)$, calculando el valor de p con un intervalo de confianza del 95\%. Se utilizó el programa Excel para realizar la base de datos y para el análisis estadístico el programa SPSS (Statistical Package for Social Sciences) versión 25.

\section{RESULTADOS}

Participaron 100 familias de pacientes pediátricos con epilepsia, encontrándose que el promedio de edad de las madres fue de 38.1 años y de los padres 39.5 años, lo que da un promedio para ambos de 38.8 años (rango: 21-64). La escolaridad predominante fue secundaria, con un 51\% (51) seguida de primaria en un $41 \%$ (41). Con respecto al estado civil, sobresalió "casado/a», con un 63\% (63), y el que se observó con menos frecuencia fue el grupo de "viudo/a», con un 4\% (4) (Tabla 1).

De acuerdo con los resultados obtenidos en el test FF-SIL, la clasificación para determinar la funcionalidad familiar, se encontró 
Tabla 1. Indicadores sociodemográficos de la población en estudio

\begin{tabular}{|l|c|c|}
\hline Escolaridad & Frecuencia de aparición & Porcentaje \\
\hline Primaria & 41 & $41 \%$ \\
\hline Secundaria & 51 & $51 \%$ \\
\hline Preparatoria & 8 & $8 \%$ \\
\hline Licenciatura & 0 & $0 \%$ \\
\hline Analfabeta & 0 & $0 \%$ \\
\hline Estado civil & Frecuencia de aparición & Porcentaje \\
\hline Casado (a) & 63 & $63 \%$ \\
\hline Soltero (a) & 24 & $24 \%$ \\
\hline Viudo (a) & 4 & $4 \%$ \\
\hline Unión libre & 9 & $9 \%$ \\
\hline
\end{tabular}

\section{DISCUSIÓN}

que el $16 \%$ (16) correspondió a familias funcionales, el 41\% (41) a moderadamente funcionales, el $41 \%$ (41) a disfuncionales y solo el 2\% (2) a severamente disfuncionales (Fig. 1).

En relación con los indicadores analizados para determinar el grado de funcionalidad familiar, los que mayor puntuación alcanzaron fueron la «afectividad», con un promedio de 7.7 ( \pm 1.75$)$, seguido de "cohesión» con 7.5 ( \pm 1.46$)$. Por el contrario, los dos indicadores con menor puntuación fueron "permeabilidad» $\mathrm{y}$ «roles», con un promedio de $5.8( \pm 1.81)$ y $5.5( \pm 1.84)$ respectivamente.

Respecto a la sobrecarga del cuidador, se observó que el 57\% (57) no presentaron ningún tipo de sobrecarga, mientras que el $31 \%$ (31) presentaron sobrecarga leve y únicamente el 12\% (12) presentaron sobrecarga intensa. Se observó que las mujeres fueron quienes presentaron mayor nivel de sobrecarga.

$\mathrm{Al}$ analizar la funcionalidad familiar con la sobrecarga del cuidador primario se encuentra que existe una asociación estadísticamente significativa, con un valor de $\mathrm{p}<$ 0.001 (Tabla 2).

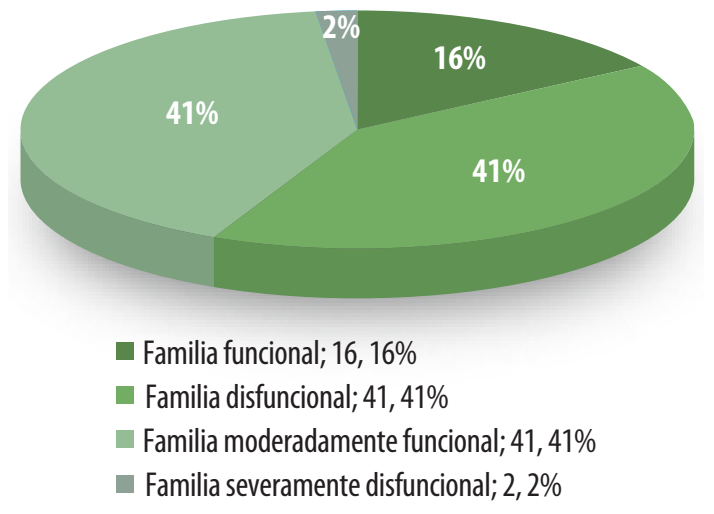

Figura 1. Clasificación de la funcionalidad y dinámica familiar.

Nuestros resultados muestran que el grupo de familias funcionales tuvo la misma cantidad de familias que el grupo de familias moderadamente funcionales. En un estudio realizado por Báez, et al. en el año 2009 en el que se evaluó la relación de sobrecarga del cuidador y el funcionamiento de las familias de niños con discapacidad motora, encontraron que el grupo más grande fueron las familias moderadamente funcionales, coincidiendo con los resultados de nuestro estudio. Al relacionar las variables de funcionalidad familiar con la sobrecarga del cuidador informal del niño que presenta discapacidad motora, se obtuvo una correlación negativa ${ }^{13}$. En nuestro estudio se confirmó la asociación estadísticamente significativa entre la funcionalidad familiar y la sobrecarga del cuidador primario.

Los indicadores en los que obtuvimos una mayor puntuación fueron «afectividad», con un promedio del 7.7\% $( \pm 1.75)$, seguido de «cohesión» con el 7.5\% $( \pm 1.46)$ y «armonía» con un 6.9\%. El indicador con menor puntuación fue «rol», con el $5.5 \%$ $( \pm 1.84)$. En otro estudio realizado por Fabelo, et al., en el cual se evaluaron las características del afrontamiento familiar a la epilepsia, los resultados mostraron que 
Tabla 2. Asociación* entre la funcionalidad familiar y la sobrecarga del cuidador primario

\begin{tabular}{|l|c|c|c|c|c|}
\hline & $\begin{array}{c}\text { Familia } \\
\text { funcional }\end{array}$ & $\begin{array}{c}\text { Familia } \\
\text { moderadamente funcional }\end{array}$ & $\begin{array}{c}\text { Familia } \\
\text { disfuncional }\end{array}$ & $\begin{array}{c}\text { Familia severamente } \\
\text { disfuncional }\end{array}$ & Total \\
\hline Sin sobrecarga & 16 & 33 & 8 & 0 & 57 \\
\hline Sobrecarga leve & 0 & 6 & 24 & 1 & 31 \\
\hline Sobrecarga intensa & 0 & 2 & 9 & 1 & 12 \\
\hline Total & 16 & 41 & 41 & 2 & 100 \\
\hline
\end{tabular}

*Prueba estadística: Chi cuadrada de Pearson; $p<0.001$.

las categorías en los procesos relacionales de mayor implicación en la disfuncionalidad familiar eran: rol, armonía y comunicación y los que se percibieron con mejores niveles de expresión eran la afectividad, la cohesión y la adaptabilidad ${ }^{14}$, lo que coincide con nuestro estudio respecto a que el indicador que mayor implicación tiene en la disfunción familiar es el rol, y el que se percibe con mejores niveles de expresión, es la afectividad. Esto se puede explicar por la tipología tradicional que hoy en día sigue predominando en las sociedades, en las que el trabajo de casa lo realiza la mujer, adoptando múltiples roles, en lugar de tener una mejor organización y distribución de estos.

\section{CONCLUSIONES}

Hay evidencia bibliográfica de varios autores que afirman que la dinámica y funcionalidad familiar se ve alterada ante la presencia de enfermedades neurológicas crónicas, como es el caso de la epilepsia, ya que se observan cambios familiares y sociales importantes, los cuales generan estrés y sobrecarga que repercuten en la vida cotidiana, ideologías y percepciones de cada uno de los integrantes de la familia.

El desafío de este estudio fue buscar la asociación entre la funcionalidad familiar y la sobrecarga del cuidador primario de pacientes pediátricos con diagnóstico de epilepsia. Los resultados nos muestran que sí existe asociación estadísticamente significativa, aceptando nuestra hipótesis de trabajo, lo cual tiene una importancia relevante para estas familias y poder brindarles atención médica integral y oportuna.

\section{AGRADECIMIENTOS}

Agradecemos al Instituto Mexicano del Seguro Social por habernos brindado la oportunidad de realizar este estudio dentro de sus instalaciones.

\section{FINANCIAMIENTO}

Para la realización del estudio no se requirió de fuente de financiamiento externo, se realizó con recursos propios de la institución.

\section{CONFLICTO DE INTERESES}

Los autores declaran no tener conflicto de intereses.

\section{RESPONSABILIDADESÉTICAS}

Protección de personas y animales. Los autores declaran que para esta investigación no se han realizado experimentos en seres humanos ni en animales.

Confidencialidad de los datos. Los autores declaran que han seguido los protocolos de su centro de trabajo sobre la publicación de datos de pacientes.

Derecho a la privacidad y consentimiento informado. Los autores declaran que en este artículo no aparecen datos de pacientes.

\section{BIBLIOGRAFÍA}

1. Vargas-Mendoza, J.E. y Sánchez-Vásquez, I. Funcionamiento familiar de pacientes pediátricos con diagnóstico de epilepsia. Centro Regional de Investigación en Psicología, Volumen 3, Número 1, 2009 Pág. 23-29. Disponible en http://www.conductitlan.net/45_ funcionamiento_familiar_pacientes_pediatricos_epilepsia.pdf 
2. Cruz-Cruz M, Gallardo Elías J, Paredes Solís S, Legorreta Soberanis J, Flores Moreno M, Andersson N. Factores asociados a epilepsia en niños en México: un estudio caso-control. Bo Med Hosp Infant Mex. 2017;4(5):334-40.

3. Epilepsia [Internet]. Organización Mundial de la Salud [citado: 10 octubre 2020]. Disponible en: https://www.who.int/topics/ epilepsy/es/

4. Fernández de Larrinoa Palacios $P$, Martínez Rodríguez $S$, Ortiz Marques N, Carrasco Zabaleta M, Solabarrieta Eizaguirre J. y Gómez Marroquín I. Autopercepción del estado de salud en familiares cuidadores y su relación con el nivel de sobrecarga. Psicothema. 2011;23(3):388-93.

5. Montero Pardo $X$ Jurado Cárdenas S, Méndez Venegas J. Variables que predicen la aparición de sobrecarga en cuidadores primarios informales de niños con cáncer. Psicooncología. 2015;12(1):67-86.

6. Marcano Caraballo R, Betancourt Henríquez F, Bravo Correa F. Síndrome de sobrecarga y grado de funcionalidad familiar en cuidadores de personas con cáncer del Hospital de Niños Rafael Tobías Guevara de Barcelona, Estado Arizoátegui, Venezuela. Biomedicina. 2017;29:394-9.

7. Pérez Peñaranda A, García Ortiz L, Rodríguez Sánchez E, Losada Baltar A, Nieves Porras S, Gómez Marcos M. Función familiar salud mental del cuidador de familiares con dependencia. Aten Primaria. 2009;41(11):621-8.
8. Ortunio Calabres M, Herrera Leonet E, Guevara Rivas H. Síndrome de carga y calidad de vida del cuidador de pacientes pediátricos. Rev Bioet Latinoam. 2016;17:1-17.

9. Martínez González L, Robles Rendón M, Ramos del Río B, Santiesteban Macario F, García Valdés M, Morales Enríquez M, et al. Carga percibida del cuidador primario del paciente con parálisis cerebral infantil severa del Centro de Rehabilitación Infantil Teletón. Rev Mex Med Fis Rehab. 2008;20:23-9.

10. Flórez Torres I, Montalvo Prieto A, Herrera Lían A, Romero Massa E. Afectación de los bienestares en cuidadores de niños y adulto con enfermedad crónica. Rev Salud Pública. 2010;12(5):754-64.

11. Ortega Veitía $T$, de la Cuesta Freijomil D, Días Retureta C. Propuesta de un instrumento para la aplicación del Proceso de Atención de Enfermería en Familias Disfuncionales. Rev Cuba Enferm. 1999:15(3):164-8.

12. Breinbauer $H$, Vásquez $H$, Mayanz $S$, Guerra $C$, Millán T. Validación en Chile de la Escala de Sobrecarga del Cuidador de Zarit en sus versiones original y abreviada. Rev Med Chile. 2009;137:657665.

13. Báez F, Medina O, Rugerio M, García G. Funcionalidad familiar y sobrecarga del cuidador del niño con discapacidad motora. Horiz Enferm. 2009;20(2):11-8.

14. Fabelo Roche J, Iglesias Moré S, Lauro Bernal I, Gonzáles Pal S Afrontamiento familiar a la epilepsia. Revista Cubana de Salud Pública. 2013;39(3):450-61. 